\title{
RECURSIVE SMOOTHERS FOR HIDDEN \\ DISCRETE-TIME MARKOV CHAINS
}

LAKHDAR AGGOUN

Received 22 September 2004 and in revised form 4 February 2005

We consider a discrete-time Markov chain observed through another Markov chain. The proposed model extends models discussed by Elliott et al. (1995). We propose improved recursive formulae to update smoothed estimates of processes related to the model. These recursive estimates are used to update the parameter of the model via the expectation maximization (EM) algorithm.

\section{Introduction}

Hidden Markov chains have been the subject of extensive studies, see the books $[1,2]$ and the references therein. Of particular interest are the discret-time, finite-state hidden Markov models.

In this paper, using the same techniques as in [3], we propose results that improve the finite-dimensional smoothers of functionals of a partially observed discrete-time Markov chain. The model itself extends models discussed in [2]. The proposed formulae for updating these quantities are recursive. Therefore, recalculation of all backward estimates is not required in the implementation of the EM algorithm.

This paper is organized as follows. In Section 2, we introduce the model. In Section 3, a new probability measure under which all processes are independent is defined and a recursive filter for the state is derived. The main results of this paper are in Section 4 where recursive smoothers are derived.

\section{Model dynamics}

A system is considered, whose state is described by a finite-state, homogeneous, discretetime Markov chain $X_{k}, k \in \mathbb{N}$. We suppose that $X_{0}$ is given, or its distribution is known. If the state space of $X_{k}$ has $N$ elements, it can be identified without loss of generality, with the set

$$
S_{X}=\left\{e_{1}, \ldots, e_{N}\right\}
$$

where $e_{i}$ are unit vectors in $\mathbb{R}^{N}$ with unity as the $i$ th element and zeros elsewhere. 
Write $\mathscr{F}_{k}^{0}=\sigma\left\{X_{0}, \ldots, X_{k}\right\}$, for the $\sigma$-field generated by $X_{0}, \ldots, X_{k}$, and $\left\{\mathscr{F}_{k}\right\}$ for the complete filtration generated by the $\mathscr{F}_{k}^{0}$; this augments $\mathscr{F}_{k}^{0}$ by including all subsets of events of probability zero. The Markov property implies here that

$$
P\left(X_{k+1}=e_{j} \mid \mathscr{F}_{k}\right)=P\left(X_{k+1}=e_{j} \mid X_{k}\right)
$$

Write $a_{j i}=P\left(X_{k+1}=e_{j} \mid X_{k}=e_{i}\right), A=\left(a_{j i}\right) \in \mathbb{R}^{N \times N}$.

Define $V_{k+1}:=X_{k+1}-A X_{k}$ so that

$$
X_{k+1}=A X_{k}+V_{k+1}
$$

$\left\{V_{k}\right\}, k \in \mathbb{N}$, is a sequence of martingale increments.

The state process $X$ is not observed directly. We observe a second Markov chain $Y$ on the same state space as $X$ but with probability transitions perturbated by $X$. More precisely, suppose that

$$
P\left(Y_{k+1}=e_{s} \mid \mathscr{G}_{k} \vee \sigma\left\{X_{k+1}\right\}\right)=P\left(Y_{k+1}=e_{s} \mid Y_{k}, X_{k+1}\right),
$$

where $\left\{\mathscr{G}_{k}\right\}$ is the complete filtration generated by $X$ and $Y$.

Write

$$
b_{s, r i}=P\left(Y_{k+1}=e_{s} \mid Y_{k}=e_{r}, X_{k+1}=e_{i}\right),
$$

and $B=\left\{b_{s, r i}\right\}, 1 \leq s, r, i \leq N$. Note that $\sum_{s=1}^{M} b_{s, r i}=1$. We immediately have the following representation for $Y$ :

$$
Y_{k+1}=B Y_{k} \otimes X_{k+1}+W_{k+1},
$$

where $W_{k}, k \in \mathbb{N}$, is a sequence of martingale increments.

Let $\left\{\mathscr{Y}_{k}\right\}$ be the complete filtration generated by $Y$.

Our objective here is to seek recursive filters and smoothers for the states of the Markov chain $X$, the number of jumps from one state to another for the occupation time of a state, and for a process related to the observations.

\section{An unnormalized finite-dimensional recursive filter for the state}

What we wish to do now is starting with a probability measure $\bar{P}$ on $\left(\Omega, \bigvee_{n=1}^{\infty} \mathscr{G}_{n}\right)$ such that

(1) the process $X$ is a finite-state Markov chain with transition matrix A;

(2) $\left\{Y_{k}\right\}, k \in \mathbb{N}$, is a sequence of i.i.d. random variables and $\bar{P}\left(Y_{k+1}=e_{r} \mid \mathscr{G}_{k} \vee \sigma\left\{X_{k+1}\right\}\right)=\bar{P}\left(Y_{k+1}=e_{r}\right)=1 / M$. 
We will now construct a new measure $P$ on $\left(\Omega, \bigvee_{n=1}^{\infty} \mathscr{G}_{n}\right)$ such that under $P, E\left[Y_{k+1} \mid\right.$ $\left.\varphi_{k} \vee \sigma\left\{X_{k+1}\right\}\right]=B Y_{k} \otimes X_{k+1}$. Write

$$
\begin{aligned}
\lambda_{\ell} & =\prod_{s, r, i=1}^{N}\left(N b_{s, r i}\right)^{\left\langle Y_{\ell}, e_{s}\right\rangle\left\langle Y_{\ell-1}, e_{r}\right\rangle\left\langle X_{\ell}, e_{i}\right\rangle}, \quad \ell \in \mathbb{N}, \\
\Lambda_{k} & =\prod_{\ell=1}^{k} \lambda_{\ell},
\end{aligned}
$$

where $b_{s, r i}$ is the probability transition defined in (2.5).

With the above definitions, $\bar{E}\left[\lambda_{k+1} \mid \mathscr{G}_{k}\right]=1$. Now set $(d P / d \bar{P}) \mid \varphi_{k}=\Lambda_{k}$. (The existence of $P$ follows from Kolmogorov's extension theorem.)

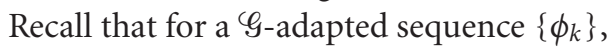

$$
E\left[\phi_{k} \mid \mathscr{Y}_{k}\right]=\frac{\bar{E}\left[\Lambda_{k} \phi_{k} \mid \mathscr{Y}_{k}\right]}{\bar{E}\left[\Lambda_{k} \mid \mathscr{Y}_{k}\right]}
$$

Write $q_{k}\left(e_{\mathfrak{m}}\right), 1 \leq t \leq N, k \in \mathbb{N}$, for the unnormalized, conditional probability distribution such that

$$
\bar{E}\left[\Lambda_{k}\left\langle X_{k}, e_{\mathfrak{m}}\right\rangle \mid \mathscr{Y}_{k}\right]=q_{k}\left(e_{\mathfrak{m}}\right) .
$$

Now $\sum_{i=1}^{N}\left\langle X_{k}, e_{i}\right\rangle=1$, so

$$
\sum_{i=1}^{N} q_{k}\left(e_{i}\right)=\bar{E}\left[\Lambda_{k} \sum_{i=1}^{N}\left\langle X_{k}, e_{i}\right\rangle \mid \mathscr{Y}_{k}\right]=\bar{E}\left[\Lambda_{k} \mid \mathscr{Y}_{k}\right]
$$

Therefore, the normalized conditional probability distribution

$$
p_{k}\left(e_{\mathfrak{m}}\right)=E\left[\left\langle X_{k}, e_{\mathfrak{m}}\right\rangle \mid \mathscr{Y}_{k}\right]
$$

is given by

$$
p_{k}\left(e_{\mathfrak{m}}\right)=\frac{q_{k}\left(e_{\mathfrak{m}}\right)}{\sum_{j=1}^{k} q_{k}\left(e_{j}\right)} .
$$

To simplify the notation, we write

$$
\begin{gathered}
c_{\mathfrak{m}}\left(Y_{k}, Y_{k-1}\right)=\prod_{s, r=1}^{N}\left(N b_{s, r \mathfrak{m}}\right)^{\left\langle Y_{k}, e_{s}\right\rangle\left\langle Y_{k-1}, e_{r}\right\rangle}, \\
\underline{c}\left(Y_{k}, Y_{k-1}\right)=\left(c_{1}\left(Y_{k}, Y_{k-1}\right), \ldots, c_{N}\left(Y_{k}, Y_{k-1}\right)\right)^{\prime} .
\end{gathered}
$$

THeOREm 3.1. For $k \in \mathbb{N}$, the recursive filter for the unnormalized estimates of the states is given by

$$
q_{k}=\operatorname{diag}\left[\underline{c}\left(Y_{k}, Y_{k-1}\right)\right] A q_{k-1}
$$


Proof. In view of (3.1), (3.2), (2.3), and the notation in (3.8),

$$
\begin{aligned}
\bar{E}\left[\Lambda_{k}\left\langle X_{k}, e_{\mathfrak{m}}\right\rangle \mid \mathscr{Y}_{k}\right] & =\prod_{s, r=1}^{N}\left(N b_{s, r \mathfrak{m}}\right)^{\left\langle Y_{k}, e_{s}\right\rangle\left\langle Y_{k-1}, e_{r}\right\rangle} \sum_{i=1}^{N} \bar{E}\left[\Lambda_{k-1}\left\langle X_{k-1}, e_{i}\right\rangle\left\langle A e_{i}, e_{\mathfrak{m}}\right\rangle \mid \mathscr{Y}_{k}\right] \\
& =c_{\mathfrak{m}}\left(Y_{k}, Y_{k-1}\right) \sum_{i=1}^{N} a_{\mathfrak{m} i}\left\langle q_{k-1}, e_{i}\right\rangle,
\end{aligned}
$$

and

$$
\begin{aligned}
\bar{E}\left[\Lambda_{k} X_{k} \mid \mathscr{Y}_{k}\right] & =\sum_{\mathfrak{m}=1}^{N} \bar{E}\left[\Lambda_{k}\left\langle X_{k} e_{\mathfrak{m}}\right\rangle \mid \mathscr{Y}_{k}\right] e_{\mathfrak{m}} \\
& =\sum_{\mathfrak{m}=1}^{N} \sum_{i=1}^{N} a_{\mathfrak{m} i} c_{\mathfrak{m}}\left(Y_{k} Y_{k-1}\right)\left\langle q_{k-1}, e_{i}\right\rangle e_{\mathfrak{m}} \\
& =\operatorname{diag}\left[\underline{c}\left(Y_{k}, Y_{k-1}\right)\right] A q_{k-1},
\end{aligned}
$$

which finishes the proof.

\section{Recursive smoothers}

We emphasize again that these improved recursive formulae to update smoothed estimates are used to update the parameters of the model via the EM algorithm.

Theorem 4.1. For $k>m$, the unnormalized smoothed estimate $\bar{E}\left[\Lambda_{k} X_{m} \mid \mathscr{Y}_{k}\right] \triangleq \gamma_{m, k}$ is given by

$$
\gamma_{m, k}=\operatorname{diag}\left[q_{m}\right] v_{m}
$$

Proof. Write $\prod_{\ell=m+1}^{k} \lambda_{\ell} \triangleq \Lambda_{m+1, k}$.

$$
\begin{aligned}
\bar{E}\left[\Lambda_{k}\left\langle X_{m}, e_{i}\right\rangle \mid \mathscr{Y}_{k}\right] & =\bar{E}\left[\Lambda_{m}\left\langle X_{m}, e_{i}\right\rangle \Lambda_{m+1, k} \mid \mathscr{Y}_{k}\right] \\
& =\bar{E}\left[\Lambda_{m}\left\langle X_{m}, e_{i}\right\rangle \bar{E}\left[\Lambda_{m+1, k} \mid \mathscr{Y}_{k} \vee \mathscr{F}_{m}\right] \mid \mathscr{Y}_{k}\right] \\
& =\bar{E}\left[\Lambda_{m}\left\langle X_{m}, e_{i}\right\rangle \bar{E}\left[\Lambda_{m+1, k} \mid \mathscr{Y}_{k} \vee \mathscr{F}_{m}\right] \mid \mathscr{Y}_{k}\right] \\
& =\bar{E}\left[\Lambda_{m}\left\langle X_{m}, e_{i}\right\rangle \bar{E}\left[\Lambda_{m+1, k} \mid \mathscr{Y}_{k} \vee\left\{X_{m}=e_{i}\right\}\right] \mid \mathscr{Y}_{k}\right] \\
& =\bar{E}\left[\Lambda_{m}\left\langle X_{m}, e_{i}\right\rangle \mid \mathscr{Y}_{m}\right] \bar{E}\left[\Lambda_{m+1, k} \mid \mathscr{Y}_{k} \vee\left\{X_{m}=e_{i}\right\}\right] \\
& \triangleq\left\langle q_{m}, e_{i}\right\rangle\left\langle v_{m}, e_{i}\right\rangle,
\end{aligned}
$$

where

$$
v_{m}=\left(\bar{E}\left[\Lambda_{m+1, k} \mid \mathscr{Y}_{k} \vee\left\{X_{m}=e_{1}\right\}\right], \ldots, \bar{E}\left[\Lambda_{m+1, k} \mid \mathscr{Y}_{k} \vee\left\{X_{m}=e_{N}\right\}\right]\right)^{\prime}
$$


Therefore,

$$
\begin{aligned}
\bar{E}\left[\Lambda_{k} X_{m} \mid \mathscr{Y}_{k}\right] & =\sum_{i=1}^{N} e_{i} \bar{E}\left[\Lambda_{k}\left\langle X_{m}, e_{i}\right\rangle \mid \mathscr{Y}_{k}\right] \\
& =\sum_{i=1}^{N}\left\langle q_{m}, e_{i}\right\rangle\left\langle v_{m}, e_{i}\right\rangle e_{i} \\
& =\operatorname{diag}\left[q_{m}\right] v_{m} .
\end{aligned}
$$

The same argument shows that the following lemma holds.

Lemma 4.2. The process $v$ satisfies the backward dynamics

$$
v_{m}=A^{*} \operatorname{diag}\left[\underline{c}\left(Y_{m+1}, Y_{m}\right)\right] v_{m+1} ; \quad v_{k}=(1, \ldots, 1) \in \mathbb{R}^{N} .
$$

Here $A^{*}$ is the matrix transpose of $A$.

4.1. Recursive smoother for the number of jumps. The number of jumps from state $e_{r}$ to state $e_{s}$ in time $k$ is given by

$$
\mathscr{F}_{k}^{r s}=\sum_{\ell=1}^{k}\left\langle X_{\ell-1}, e_{r}\right\rangle\left\langle X_{\ell}, e_{s}\right\rangle .
$$

Theorem 4.3. Write $\sigma\left(\mathscr{F}_{k}^{r s}\right)=\bar{E}\left[\Lambda_{k} \mathscr{F}_{k}^{r s} \mid \mathscr{Y}_{k}\right]$.

$$
\sigma\left(\mathscr{F}_{k}^{r s}\right)=\sum_{\ell=1}^{k} a_{s r}\left\langle q_{\ell-1}, e_{r}\right\rangle\left\langle v_{\ell-1}, e_{r}\right\rangle .
$$

Proof.

$$
\begin{aligned}
\bar{E}\left[\Lambda_{k} \mathscr{J}_{k}^{r s} \mid \mathscr{Y}_{k}\right] & =\sum_{\ell=1}^{k} \bar{E}\left[\left\langle X_{\ell-1}, e_{r}\right\rangle\left\langle X_{\ell}, e_{s}\right\rangle \Lambda_{k} \mid \mathscr{Y}_{k}\right] \\
& =\sum_{\ell=1}^{k} \bar{E}\left[\left\langle X_{\ell-1}, e_{r}\right\rangle\left\langle A X_{\ell-1}, e_{s}\right\rangle \Lambda_{k} \mid \mathscr{Y}_{k}\right] \\
& =\sum_{\ell=1}^{k} a_{s r} \bar{E}\left[\left\langle X_{\ell-1}, e_{r}\right\rangle \Lambda_{k} \mid \mathscr{Y}_{k}\right] \\
& =a_{s r} \sum_{\ell=1}^{k} \bar{E}\left[\Lambda_{\ell-1}\left\langle X_{\ell-1}, e_{r}\right\rangle \bar{E}\left[\Lambda_{\ell, k} \mid \mathscr{Y}_{k} \vee\left\{X_{\ell-1}=e_{r}\right\}\right] \mid \mathscr{Y}_{k}\right] \\
& =a_{s r} \sum_{\ell=1}^{k}\left\langle q_{\ell-1}, e_{r}\right\rangle\left\langle v_{\ell-1}, e_{r}\right\rangle,
\end{aligned}
$$

which finishes the proof. 
LeMma 4.4.

$$
\sigma\left(\mathscr{F}_{k+1}^{r s}\right)=\Gamma_{k}^{\prime} A^{*} \operatorname{diag}\left[\underline{c}\left(Y_{k+1}, Y_{k}\right)\right] \cdot \underline{1}+a_{s r}\left\langle q_{k}, e_{r}\right\rangle,
$$

where $\underline{1}=(1, \ldots, 1) \in \mathbb{R}^{N}$ and

$$
\Gamma_{k}^{\prime} \triangleq a_{s r} \sum_{\ell=1}^{k}\left\langle q_{\ell-1}, e_{r}\right\rangle e_{r}^{\prime} A^{*} \operatorname{diag}\left[\underline{c}\left(Y_{\ell}, Y_{\ell-1}\right)\right] \cdots A^{*} \operatorname{diag}\left[\underline{c}\left(Y_{k-1}, Y_{k}\right)\right]
$$

Proof. Using the backward recursion (4.5),

$$
\begin{aligned}
\sigma\left(\mathscr{F}_{k}^{r s}\right) & =a_{s r} \sum_{\ell=1}^{k}\left\langle q_{\ell-1}, e_{r}\right\rangle e_{r}^{\prime} A^{*} \operatorname{diag}\left[\underline{c}\left(Y_{\ell}, Y_{\ell-1}\right)\right] \cdots A^{*} \operatorname{diag}\left[\underline{c}\left(Y_{k+1}, Y_{k}\right)\right] \cdot \underline{1} \\
& =\Gamma_{k}^{\prime} \cdot \underline{1} .
\end{aligned}
$$

Also note that

$$
\Gamma_{k+1}^{\prime}=\Gamma_{k}^{\prime} A^{*} \operatorname{diag}\left[\underline{c}\left(Y_{k+1}, Y_{k}\right)\right]+a_{s r}\left\langle q_{k}, e_{r}\right\rangle e_{r}
$$

Therefore,

$$
\sigma\left(\mathscr{F}_{k+1}^{r s}\right)=\Gamma_{k+1}^{\prime} \cdot \underline{1}=\Gamma_{k}^{\prime} A^{*} \operatorname{diag}\left[\underline{c}\left(Y_{k+1}, Y_{k}\right)\right] \cdot \underline{1}+a_{s r}\left\langle q_{k}, e_{r}\right\rangle,
$$

and the result follows.

4.2. Recursive smoother for the occupation time. The number of occasions up to time $k$ for which the Markov chain $X$ has been in state $e_{r}, 1 \leq r \leq N$, is

$$
\mathrm{O}_{k}^{r}=\sum_{\ell=0}^{k}\left\langle X_{\ell}, e_{r}\right\rangle
$$

Lemma 4.5. Write $\sigma\left(\bigcirc_{k}^{r}\right)=\bar{E}\left[\Lambda_{k} \bigcirc_{k}^{r} \mid \mathscr{Y}_{k}\right]$.

$$
\begin{gathered}
\sigma\left(\mathcal{O}_{k}^{r}\right)=\sum_{\ell=0}^{k}\left\langle q_{\ell}, e_{r}\right\rangle\left\langle v_{\ell}, e_{r}\right\rangle, \\
\sigma\left(\mathcal{O}_{k+1}^{r}\right)=\sum_{k}^{\prime} A^{*} \operatorname{diag}\left[\underline{c}\left(Y_{k+1}, Y_{k}\right)\right] \cdot \underline{1}+\left\langle q_{k+1}, e_{r}\right\rangle,
\end{gathered}
$$

where

$$
\Sigma_{k}^{\prime} \triangleq \sum_{\ell=1}^{k}\left\langle q_{\ell}, e_{r}\right\rangle e_{r}^{\prime} A^{*} \operatorname{diag}\left[\underline{c}\left(Y_{\ell}, Y_{\ell-1}\right)\right] \cdots A^{*} \operatorname{diag}\left[\underline{c}\left(Y_{k-1}, Y_{k}\right)\right]
$$

and

$$
\Sigma_{k+1}^{\prime}=\Sigma_{k}^{\prime} A^{*} \operatorname{diag}\left[\underline{c}\left(Y_{k+1}, Y_{k}\right)\right]+\left\langle q_{k+1}, e_{r}\right\rangle e_{r} .
$$


4.3. Recursive smoother for state-to-observation transitions. The parameters estimation of our model requires estimates and smoothers of the process

$$
\mathscr{T}_{k}^{r s \mathfrak{m}}=\sum_{\ell=1}^{k}\left\langle X_{\ell-1}, e_{r}\right\rangle\left\langle Y_{\ell-1}, e_{s}\right\rangle\left\langle Y_{\ell}, e_{\mathfrak{m}}\right\rangle
$$

Lemma 4.6. Write $\sigma\left(\mathscr{T}_{k}^{r s m}\right)=\bar{E}\left[\Lambda_{k} \mathscr{T}_{k}^{r s} \mid \mathscr{Y}_{k}\right]$

$$
\begin{aligned}
\sigma\left(\mathscr{T}_{k}^{r s}\right) & =\sum_{\ell=1}^{k}\left\langle q_{\ell-1}, e_{r}\right\rangle\left\langle v_{\ell-1}, e_{r}\right\rangle\left\langle Y_{\ell-1}, e_{s}\right\rangle\left\langle Y_{\ell}, e_{\mathfrak{m}}\right\rangle, \\
\sigma\left(\mathscr{T}_{k+1}^{r s \mathfrak{m}}\right) & =\Phi_{k}^{\prime} A^{*} \operatorname{diag}\left[\underline{c}\left(Y_{k+1}\right)\right] \cdot \underline{1}+\left\langle q_{k}, e_{r}\right\rangle\left\langle Y_{k+1}, e_{s}\right\rangle,
\end{aligned}
$$

where

$$
\Phi_{k}^{\prime} \triangleq \sum_{\ell=1}^{k}\left\langle q_{\ell-1}, e_{r}\right\rangle\left\langle Y_{\ell-1}, e_{s}\right\rangle\left\langle Y_{\ell}, e_{\mathfrak{m}}\right\rangle e_{r}^{\prime} A^{*} \operatorname{diag}\left[\underline{c}\left(Y_{\ell}\right)\right] \cdots A^{*} \operatorname{diag}\left[\underline{c}\left(Y_{k}\right)\right],
$$

and

$$
\Phi_{k+1}^{\prime}=\Phi_{k}^{\prime} A^{*} \operatorname{diag}\left[\underline{c}\left(Y_{k+1}\right)\right]+\left\langle q_{k}, e_{r}\right\rangle\left\langle Y_{\ell-1}, e_{s}\right\rangle\left\langle Y_{\ell}, e_{\mathfrak{m}}\right\rangle e_{r}
$$

\section{References}

[1] L. Aggoun and R. J. Elliott, Measure Theory and Filtering: Introduction and Applications, Cambridge Series in Statistical and Probabilistic Mathematics, Cambridge University Press, Cambridge, 2004.

[2] R. J. Elliott, L. Aggoun, and J. B. Moore, Hidden Markov Models: Estimation and Control, Applications of Mathematics (New York), vol. 29, Springer-Verlag, New York, 1995.

[3] R. J. Elliott and W. P. Malcolm, Improved smoother dynamics for discrete-time HMM parameter estimation, Proceeding of the 40th IEEE Conference on Decision and Control, Florida, 2001.

Lakhdar Aggoun: Department of Mathematics and Statistics, Sultan Qaboos University, P.O. Box 36, Al-Khodh 123, Muscat, Oman

E-mail address: laggoun@squ.edu.om 


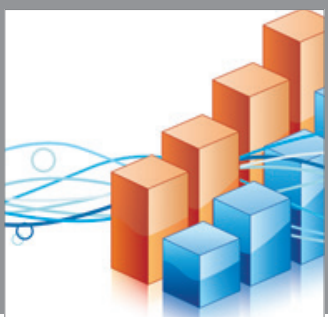

Advances in

Operations Research

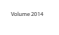

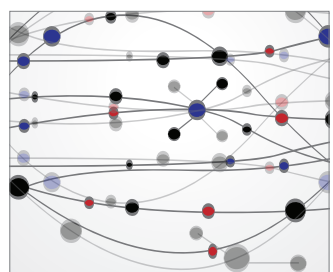

\section{The Scientific} World Journal
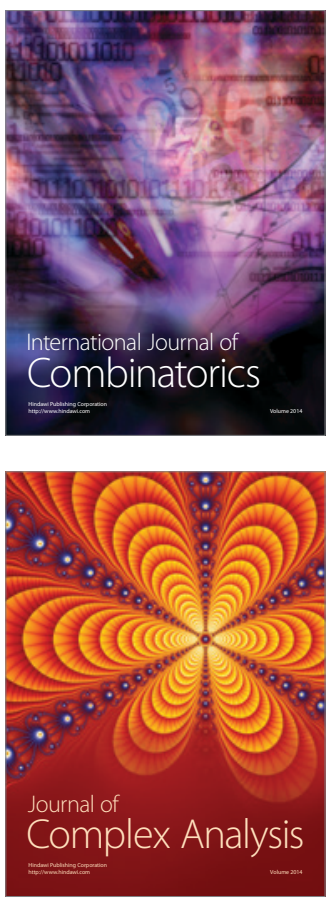

International Journal of

Mathematics and

Mathematical

Sciences
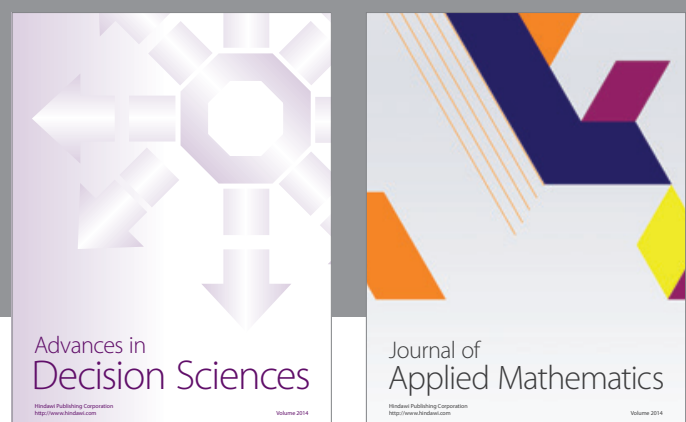

Journal of

Applied Mathematics
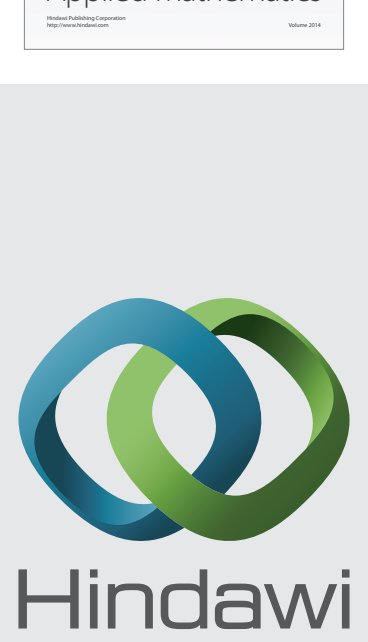

Submit your manuscripts at http://www.hindawi.com
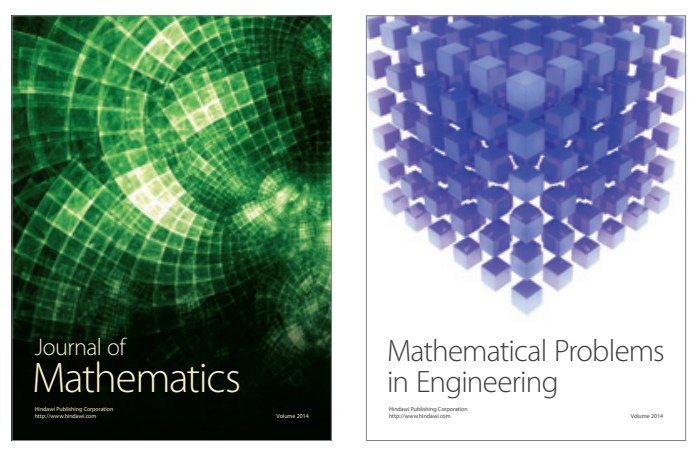

Mathematical Problems in Engineering
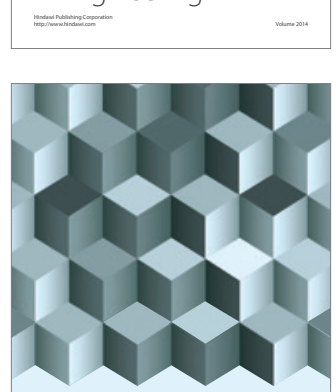

Journal of

Function Spaces
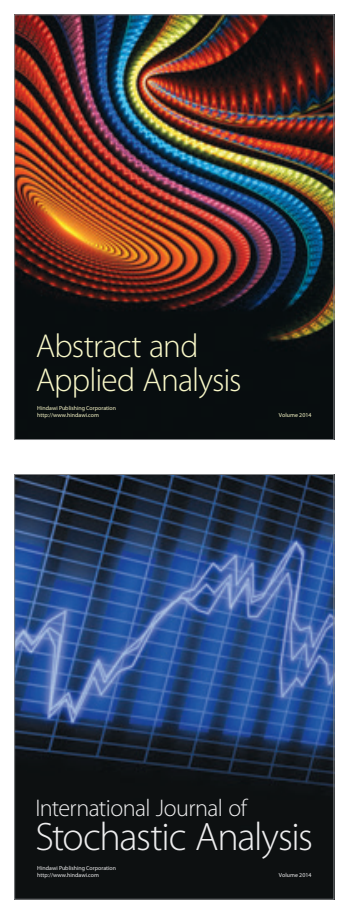

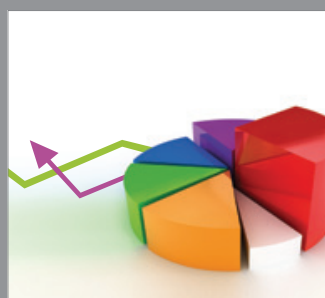

ournal of

Probability and Statistics

Promensencen
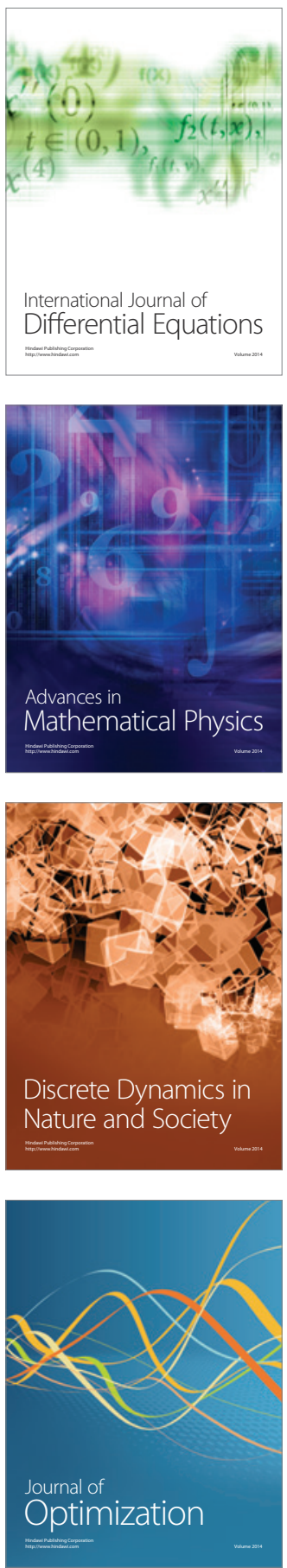